\title{
An examination of a proposed DSM-IV Pathological Gambling Hierarchy \\ in a Treatment Seeking Population: Similarities with substance dependence and evidence for three classification systems
}

Darren R. Christensen, ${ }^{1,7}$, Alun C. Jackson ${ }^{1,4}$, Nicki A. Dowling ${ }^{1,5,6}$, Rachel A. Volberg ${ }^{2}$, and Shane Thomas ${ }^{3}$

${ }^{1}$ Problem Gambling Research and Treatment Centre, University of Melbourne

${ }^{2}$ Gemini Research

${ }^{3}$ Problem Gambling Research and Treatment Centre, Monash University

${ }^{4}$ Heart Research Centre

${ }^{5}$ School of Psychiatry and Psychology, Monash University

${ }^{6}$ School of Psychology, Deakin University

${ }^{7}$ Faculty of Health Sciences, University of Lethbridge

Correspondence concerning this article should be addressed to:

Darren R. Christensen,

Faculty of Health Sciences,

University of Lethbridge,

4401 University Drive West,

Lethbridge, AB T1K 3M4

Canada

Email: darren.christensen@uleth.ca

Co-author contact information:

Alun C. Jackson: aluncj@unimelb.edu.au

Nicki A. Dowling: nicki.dowling@deakin.edu.au

Rachel A. Volberg: rvolberg@geminiresearch.com

Shane Thomas: shane.thomas@monash.edu 
Toce-Gerstein et al. (2003) investigated the distribution of DSM-IV (Diagnostic and Statistical Manual for Mental Disorders, $4^{\text {th }}$ edition) pathological gambling criteria endorsement in a U.S. community sample for those people endorsing a least one of the DSM-IV criteria ( $\mathrm{n}=399)$. They proposed a hierarchy of gambling disorders where endorsement of 1-2 criteria were deemed 'At-Risk', 3-4 'Problem gamblers’, 5-7 'Low Pathological’, and 8-10 ‘High Pathological' gamblers. This article examines these claims in a larger Australian treatment seeking population. Data from 4349 clients attending specialist problem gambling services were assessed for meeting the ten DSM-IV pathological gambling criteria. Results found higher overall criteria endorsement frequencies, three components, a direct relationship between criteria endorsement and gambling severity, clustering of criteria similar to the ToceGerstein et al. taxonomy, high accuracy scores for numerical and criteria specific taxonomies, and also high accuracy scores for dichotomous pathological gambling diagnoses. These results suggest significant complexities in the frequencies of criteria reports and relationships between criteria. 
Pathological gambling became recognised as a psychiatric disorder when it was first introduced to the Diagnostic and Statistical Manual in the third edition (American Psychiatric Association, 1980). In the DSM-IV TR edition (American Psychiatric Association, 2000) pathological gambling was classified under 'Impulse-Control Disorders Not Elsewhere Classified,' along with compulsive stealing (kleptomania), fire starting (pyromania) and hair pulling (trichotillomania). The DSM-V Work Group has renamed pathological gambling 'disordered gambling' and moved it under a new classification titled 'Substance Related and Addictive Disorders'. Disordered gambling, which is the sole 'behavioral addiction' in this group, is thought to have similarities to cognitive, neurological, genetic, and behavioural relationships found in substance dependence (Cavedini, Riboldi, Keller, D'Annucci, \& Bellodi, 2002; de Ruiter et al., 2009; Goudriaan, Oosterlaan, de Beurs, \& van den Brink, 2006; Slutske et al., 2003), as well as high rates of substance dependence comorbidity (Lorains, Cowlishaw, \& Thomas, 2011; Welte, Barnes, Wieczorek, Tidwell, \& Parker, 2001).

Regardless of the specific conceptual model employed, and the terms used, problematic gambling has become associated with dichotomous classificatory systems such as the DSM-IV and the South Oaks Gambling Screen (Lesieur, 2006) which establish 'case-ness' by meeting a standard cut-off score. A second approach has been to define gambling issues along a continuum of gambling-related harm where increasing criteria endorsement indicates increasing severity (Ferris \& Wynne, 2001).

More recently, a third suggestion has also been made, that there may be a hierarchy of gambling symptoms across the gambling severity continuum (Toce-Gerstein, Gerstein, \& Volberg, 2003). This third possibility has important implications as it raises the possibility of attributing specific DSM-IV criteria to specific gambling severities, creating a more nuanced 
understanding of severity. Moreover, a specified criteria based-hierarchy may produce a qualitatively different phenomenology of harm and better predictive accuracy than a system based on increasing numbers of endorsed criteria. Further, some research suggests that the diagnostic accuracy of the DSM 'case-ness' approach can be improved if some criteria are given greater value than other criteria. For example, Stinchfield et al. (2005) found four criteria: preoccupation, loss of control, gambling to escape, and chasing losses were more powerful predictors across a range of measures than other criteria for discriminating between disordered and non-disorders groups. Moreover, Stinchfield et al. (2005) found that using all of the criteria in a Discriminant Function Analysis was a better predictor of classification accuracy than either a cut-off score of four or five endorsed criteria. Such results may support a hierarchical structure in the valuing of criteria and question the appropriateness of using only 'case-ness' as the sole diagnostic approach.

Toce-Gerstein et al. (2003) attempted to refine the diagnosis of pathological gambling by examining the association of individual DSM-IV diagnostic criteria relative to the distribution of gamblers from a general population survey and an in-person survey of gambling facility patrons in the United States. The combined sample from these two studies comprised 399 participants who reported at least one loss of more than $\$ 100$ in a single year and endorsed one or more DSM-IV symptoms of life time pathological gambling. These scores were then categorized based on the National Opinion Research Centre Diagnostic Screen for Gambling Problems (NODS) taxonomy, and suggested four levels of problem gambling severity: a) At-Risk gamblers reporting predominantly Chasing gambling losses, gambling Preoccupation, and gambling for emotional Escape; b) Problem gamblers endorsing elevated rates of Chasing gambling losses, Preoccupation with gambling, gambling for 
emotional Escape, and Lying to others; c) Low Pathological gamblers endorsing elevated rates of Loss of Control, Withdrawal symptoms, Tolerance, Risked Relationships and needing to be Bailed out financially from gambling debts or paying for essential goods and services; and d) High Pathological gamblers endorsing elevated rates for almost all of the ten DSM-IV criteria and higher endorsement rates of Illegal Acts than any other gambling severity.

Toce-Gerstein et al. (2003) argued that although the severity of gambling problems can be represented along a continuum, their results suggested that groupings of symptoms may also be predictive of four separate patterns; an At-Risk non-clinical pattern marked by Chasing losses, a Problem subclinical pattern, a clearly separate diagnosis of Low Pathological gambling, and a more severe level of High Pathological gambling marked by elevated rates of Illegal Acts. They also suggested this hierarchy can make important clinical distinctions between sub-clinical problem gambling and pathological gambling based on the endorsement of Withdrawal and Loss of Control, as these criteria both describe the phenomenon of repeated and unsuccessful attempts to resist the urge to gamble; a phenomenon typically associated with problematic gambling (Kushner et al., 2007). Further, similar sub-clinical distinctions have been indicated in other community samples, notably Chasing in both the 2007 British Gambling Prevalence Study (Orford, Wardle, Griffiths, Sproston, \& Erens, 2010), and also by U.S. young adults in the National Epidemiological Study of Alcohol and Related Conditions (Strong \& Kahler, 2007).

Although Toce-Gerstein et al. provided some initial analyses based on frequencies of criteria endorsement and a possible 'sub-clinical' item identified in the principal component analysis, no evidence was provided for their proposed hierarchy. Specifically, neither the specifications of which symptoms belong to which level in the hierarchy, nor evidence 
supporting the proposed cut-points was given. Moreover, their taxonomy was based on the frequency of criteria endorsement rather than distinct criteria groupings. This approach is somewhat different from the typical understanding of taxa: qualitative distinctions between groups (Shaffer \& Martin, 2011). Examining these issues will provide a broader context with which to view the Toce-Gerstein et al. hierarchy and perhaps make new distinctions between treatment and non-treatment populations.

\begin{abstract}
Aims
The present study attempts to address these issues by investigating whether a similar hierarchy of DSM-IV criteria in an Australian treatment seeking sample exists when assessments are made by specialist problem gambling counselors. The Toce-Gerstein et al. analyses will be replicated and their proposed hierarchy will be tested using new analyses.
\end{abstract}

\title{
Hypotheses
}

The hypotheses for this study are; 1) as treatment seekers are experiencing more harm than those not seeking treatment their DSM-IV criteria endorsement will be higher than the Toce-Gerstein et al. community sample, 2) as pathological gambling appears to share similarities with substance dependence we expect the clustering of DSM-IV pathological gambling criteria to reflect the physiological responses of substance dependence, i.e. significant associations between Tolerance and Withdrawal, and 3) as there is some support for different gambling severity classification systems we expect the three systems mentioned earlier (dichotomous 'case-ness', continuum, and hierarchical) to each be predictive of gambling criteria endorsement. 
Gambling Disorder Hierarchy

\section{Method}

\section{Participants}

The dataset consisted of 4349 co-registered (matched registration and assessment) records where at least one DSM-IV criterion had been met, and the age of the client was reported as 18 years or more. All clients were treatment seekers, although it is not known whether participants had any previous treatment for their gambling. The current study's sample comprised of 2144 females and 2125 males (80 unreported gender), aged from 18 to 86 years $(\mathrm{M}=38.44, \mathrm{SD}=11.04$, median $=37)$. The majority of the sample was born in Australia (75\%), employed for less than 35 hours per week (62\%), and were not in a current relationship (53\%). The amount of gambling-related debt for this sample ranged from AUS\$0 to $\$ 2$ million $(\mathrm{M}=\$ 10653, \mathrm{SD}=53944$, median $=\$ 1500)$.

\section{Procedure}

The data were collected in the first treatment session post-intake by Gambler’s Help (Victoria) problem gambling clinicians using semi-structured clinical interviews. No interviews were recorded. This service was funded at the time of the study by the Victorian Department of Human Services (now funded by the Victorian Responsible Gambling Foundation) and implemented by various counseling agencies to provide specialist problem gambling counseling free of charge to clients across approximately 100 sites (urban and rural) in the state of Victoria, Australia. Two of the authors in the present study managed the dataset as part of an evaluation of Victorian Gambler's Help services and reported these findings to 
the Department of Human Services. Counselors regularly sent de-identified information to these authors who periodically reviewed reporting procedures. Clients were notified that their de-identified summary data would be reported to the Victorian Government and presented in publicly available documents. Missing criteria endorsement information was rigorously followed up by research assistants resulting in $99 \%$ of cases entered into the dataset. The current analysis was approved by the University of Melbourne Human Research Ethics Committee (Approval number 980115).

\section{DSM-IV Criteria}

Data were sourced from an archived data set comprising registration data and assessment data collected from clients seeking treatment for their gambling problems attending the Victorian Gamblers Help services from 1997 to 2002. Two separate datasets, one comprising client registration data and the other client assessment data, were merged to form the current dataset. Counselors followed a semi-structured clinical interview process where counselors were directed to assess participants on each DSM-IV pathological gambling criteria for current pathological gambling and record either endorsement or absence. No set language was prescribed for making these assessments but the DSM-IV criteria were listed and the counselors were asked if these statements 'typify your clients' actions' at the time of presentation. This was a common approach for diagnoses in clinical settings at the time with (Ladouceur, Silvain, Boutin, \& Doucet, 1998) for example, suggesting that 'determining whether a criterion is met sometimes meets with ambiguity or difficulties. In such cases, it is helpful to reformulate the question in such a way as to obtain a clear and precise response' (p41). Moreover, using a brief check-list approach, as used in this study, was the then 
assessment method of choice in treatment settings (Neal, Delfrabbro, \& O'Neil, 2005). Typically, this involved the clinician probing the client using open-ended questions based on the language and contingencies found in the DSM-IV criteria until the clinician was satisfied they could make a diagnostic evaluation on each criterion. For example, the Withdrawal criterion might be probed by asking 'can you tell me about how you have felt when you have tried to cut-down or stop gambling?', while the Tolerance might be probed by asking 'can you tell me about your gambling expenditure these past few weeks?' and then by asking 'can you explain why you needed to spend this amount of money?'. Counselors were given specific training on the DSM-IV criteria for pathological gambling in a single session and were provided with a manual describing the assessment and registration process but no measure of training effectiveness was undertaken. The client registration form described the general demographic variables while the client assessment form included the DSM IV criteria, gambling behavior, and amounts of money spent during a typical gambling episode. No reports of co-morbid substance abuse, duration of gambling disorder, or treatment outcomes were available for analysis.

\section{Counselor Information}

Data were collected by 49 counselors working in 18 different agencies in the state-wide problem gambling counseling service. Approximately $66 \%$ of the counselors were female. Overall, the mean age for both male and female counselors was 40.5 years. A little over twothirds of the sample was qualified as either social workers or psychologists. Counsellors with these qualifications were primarily located in metropolitan agencies. Other qualifications which counselors held were welfare studies (14\%) while the remaining one-fifth of the 
counselors were qualified in a range of areas including occupational therapy, psychiatric nursing, education, childcare and social science generalist degrees. These 'other qualified' were located primarily in rural agencies. Additionally, a majority of counselors (87\%) had attended specialist training courses in problem gambling counseling, although no information was available regarding their counseling or specific gambling counseling experience. Half of the gambling counseling programs were located within Community Health Centres, with another $28 \%$ located within family support agencies. The remaining programs were located within agencies primarily involved in the delivery of material aid (11\%) and drug and alcohol counseling (11\%).

\section{Data analysis}

As a starting point, we replicated the Toce-Gerstein et al. analyses as much as our data would allow. Initially, frequency distributions were conducted to compare our results with those from Toce-Gerstein et al. to test the hypothesis 1 prediction that treatment seekers will have higher endorsement rates of DSM-IV criteria than those from the community sample. The following frequencies were calculated; the proportion of participants meeting each DSMIV criterion, the frequency of participants meeting the Toce-Gerstein et al. subgroup cut-off scores, and the proportion of participants reporting DSM-IV criteria in each subgroup.

We then classified treatment seekers using the Toce-Gerstein et al. methodology. Specifically, gamblers were categorized as: At-Risk (i.e., those who met one-two DSM-IV criteria), Problem (i.e., those who met three-four DSM-IV criteria), Low Pathological (i.e., those who met five-seven DSM-IV criteria), and High Pathological (i.e., those who met eight10 DSM-IV criteria). Pair-wise comparisons were then made for each subgroup and for the 
sample as a whole using Fisher's exact tests reporting Cramer's V associations. These analyses assessed the hypothesis 2 prediction that the physiological criteria indicative of substance dependence, i.e. Tolerance and Withdrawal, are more highly correlated with each other than other criteria in a gambling treatment seeking sample. We only report significant pair-wise associations at .3 magnitude or above.

A Principal Component Analysis (PCA) was run to investigate the associations between criteria and statistically constructed combinations of groups of DSM-IV criteria (components). The component where the largest loading for each criterion fell was taken as evidence of that criterion belonging to that component. Unusually, Toce-Gerstein et al. did not rotate components when they ran their principal component analysis. This approach does not maximize the component loadings making interpretations on the relative strength of the DSMIV criteria within components questionable. Consequently, we obliquely rotated criteria using the Promax method with Kaiser normalization for more accurate criteria loadings. This analysis also contributed to the examination of the hypothesis 2 prediction that physiological criteria are likely to associate together.

Further, univariate curves were plotted for each separate DSM-IV criterion for the complete range of non-zero DSM-IV scores where linear and polynomial curves were fitted to the observed plots maximizing the squared correlation between observed and predicted values. The best fitting curve to two decimal places was then chosen as representative of the underlying functions within the data (as specified by Toce-Gerstein et al. (2003); a decelerating curvilinear concave downwards curve indicated a 'low-threshold' relationship between the criterion and gambling severity, a linear curve indicated a directly dependent relationship, and an accelerating concave upwards curve indicated a 'high-threshold' 
relationship). Polynomial curves were fitted to the data using three or four free parameters, depending if the additional free parameter added to the fit of the squared correlations.

Additionally, to quantify which type of curve best describes the data, the Akaike Information Criterion (Akaike, 1974) was used to compare which function was the best estimate of the 'true model' based on the complexity and the goodness of fit of the univariate curves to the data. An analysis of the best-fitting curves contributes to our examination of the hypothesis 3 prediction; that there will be evidence of linear relationships (a continuum of severity) between the percentage of criterion endorsement and total DSM-IV scores (dichotomous and hierarchical classification systems will be examined in the cluster and discriminant analyses). In addition to the original Toce-Gerstein et al. analyses, hierarchical cluster analyses of the DSM-IV criteria were conducted to examine the clustering and order of criteria. Squared euclidean distances between criteria were calculated to form clusters that were step-wise amalgamated together at increasingly larger distances. This provides a hierarchical schedule of clustering showing the order and distances between criteria and clusters in the amalgamation process. The cluster analyses investigates hypothesis 2 ; that physiological criteria are likely to associate together in the same cluster, and contributes to our testing of the hypothesis 3 by assessing for the existence of a hierarchy of criteria endorsement.

Discriminant analyses were run to examine hypothesis 3; calculating the predictive accuracy of three competing classification systems (dichotomous, continuum, hierarchical). Several analyses were run. We first assessed the predictive accuracy of the original ToceGerstein et al. hierarchy using numerical cut-off scores; 1-2, 3-4, 5-7, and 8-10. Next, based on the accuracy scores for each sub-group, we investigated variations of the original taxonomy. We also assessed the predictive ability of the best performing hierarchical cluster cut-offs 
based on specific criteria endorsement. Finally, we ran an analysis using the DSM-IV 'caseness' cut-off score (endorsement of five or more criteria) and an analysis of the predictive accuracy of the DSM-V cut-off score (four or more criteria, excluding Illegal Acts). All analyses were run using separate group covariance matrices.

The main statistical analyses were conducted using SPSS 19.0@ except for the model fitting procedure which was run using the Excel $\odot$ solver data analysis application. Additional statistical comparisons between the current study and Toce-Gerstein et al. were made using the online calculator from the Answers Research website. An archived link to the web page is available here: http://www.webcitation.org/67tDV3q5Z. Missing data were excluded casewise from the analyses.

\section{Results}

\section{DSM-IV Criteria}

The proportion of participants meeting each DSM-IV criterion is displayed in Table 1. Examination of Table 1 reveals that the current study sample meets all of the DSM-IV criteria more frequently than in the earlier community study (hypothesis 1 prediction; supported). Table 1 also shows that the most commonly met criteria in the current study were, in order: Escape, Chasing, Lying, and Loss of Control.

\section{--- INSERT TABLE 1 ABOUT HERE ---}

\section{Taxonomy}

Counselors reported their clients had much higher percentages of criteria endorsement in 
the Low Pathological and High Pathological sub-groups (see Table 2), while a much smaller proportion was classified as At-Risk gamblers in the current study compared to Toce-Gerstein et al. (2003). As a consequence, only the Problem Gambler subgroup proportions were nonsignificant at the 95\% confidence level when the current study was compared with the ToceGerstein et al. (2003) study.

\section{--- INSERT TABLE 2 ABOUT HERE ---}

Table 3 displays the proportion of participants reporting each DSM-IV criterion using the Toce-Gerstein et al. (2003) taxonomy. All differences between subgroups were statistically significant except for the Illegal Acts criterion between the At-Risk and Problem Gambler sub-groups. The average number of criteria endorsed was 6.28 (median 6.00, standard deviation 2.27).

\section{--- INSERT TABLE 3 ABOUT HERE ---}

Table 4 shows the pair-wise associations between criteria for the entire study. All criteria except for Illegal Acts were significantly associated with all other criteria. Illegal Acts had significant associations with all but two of the other criteria: Withdrawal and Loss of Control. The strongest association across all criteria was between Preoccupation and Tolerance (hypothesis 2 prediction; partially supported - although there were strong associations between Withdrawal and Tolerance, there were stronger associations between other pairs of criteria). 
Gambling Disorder Hierarchy

--- INSERT TABLE 4 ABOUT HERE ---

\section{Taxonomy Subgroups}

Although Toce-Gerstein et al. (2003) stated their sample size was insufficient to support a separate analysis for individual subgroups, the present study had substantially more participants (Toce-Gerstein et al. (2003): $n=399$, Current study: $n=4349$ ). Thus, in our analyses of individual taxonomies, the most frequently associated DSM-IV criteria within subgroups are also reported. For each subgroup; proportion of total sample, relative DSM-IV criteria frequencies between adjacent subgroups, comparisons between the DSM-IV criteria and Toce-Gerstein et al. (2003) taxonomy frequencies, and the highest degree of association between DSM-IV criteria within each subgroup are reported.

At-Risk gambling. Table 2 showed that At-Risk gamblers comprised 7\% of the sample of gamblers presenting to specialist problem gambling services. Only Escape (61.2\%) was reported by more than $50 \%$ of the At-Risk gamblers in the sample (See Table 3). When examining At-Risk gamblers only, Escape and Risked Relationships were found to be the strongest associated criteria (.38), as well as Escape and Illegal Acts (.37), while Risked Relationships and Illegal Acts was also significant (.33). Given the high reports of Escape and the strong connection to other criteria, Escape appears to be a central experience for At-Risk gamblers presenting to specialist services.

Problem gambling. Table 2 showed problem gamblers comprised 16\% of our sample. Table 3 showed the proportion of gamblers reporting each criterion significantly increased between At-Risk and Problem gambling for most criteria. Only Escape (78.5\%), Chasing 
(55.6\%), and Lying (50.9\%) were reported by 50\% or more of Problem gamblers. When examining Problem gamblers, Lying and Loss of Control was the only significant pair-wise association at or above the magnitude cut-off (.30).

Low Pathological gambling. Table 2 showed Low Pathological gamblers comprised $44 \%$ of our sample. Table 3 showed the proportion of gamblers reporting each criterion significantly increased between Problem gambling and Low Pathological gambling. Table 3 showed that most criteria were reported by 50\% of Low Pathological gamblers; Escape (86.8\%), Chasing (85.4\%), Lying (80.2\%), Loss of Control (76.6\%), Preoccupation (61.8\%), Bailout (52.3\%), and Withdrawal (52.2\%). When examining Low Pathological gamblers only, Risked Relationships and Preoccupation had the strongest association (.35) while Risked Relationships and Tolerance had the second strongest (.33). Bailout and Withdrawal was also significant (.31), as were Withdrawal and Risked Relationships, Bailout and Tolerance, and Preoccupation and Tolerance, all at the magnitude cut-off (.30). Given that Low Pathological gamblers met Risked Relationships almost twice as much as Problem gamblers, the increase in severity appears related to an increase in the number of issues reported by participants in their relationships.

High Pathological gambling. Table 2 showed that High Pathological gamblers comprised 32\% of our sample. Table 3 showed the proportion of gamblers reporting each criterion significantly increased between Low and High Pathological gambling. High Pathological gamblers met most criteria at a consistently high frequency; Chasing (98.3\%), Escape (97.2), Lying (97.2\%), Loss of Control (96.1\%), Preoccupation (92.5\%), Withdrawal (91.8\%), Bailout (89.2\%), Tolerance (87.8), and Risked Relationships (86.8\%). When examining High Pathological gamblers only, although there were several significant 
associations, none met the magnitude cut-off score (.30). Despite Illegal Acts being the least frequently met criteria it had the greatest number of associations with other criteria, supporting a previous finding that gamblers who are committing crimes are experiencing the greatest degree of problem gambling severity (Strong \& Kahler, 2007).

Subgroup Comparisons. Comparing the number of criteria met across sub-groups (Table 3), there appears to be a separation in the number of criteria that at least $50 \%$ of participants met between the At-Risk and the Problem gambler groups (1 and 3 criteria, respectively) compared with the numbers met by Low Pathological and High Pathological gambler groups (7 and 9 criteria, respectively). However, this dichotomy is not reflected in the sub-group pair-wise association between criteria where no discernible pattern exists.

\section{Principal Component Analyses}

A Principal Component Analysis (PCA) found three components to have Eigen values greater than $1.0(2.628,1.475$, and 1.050 for components 1 , 2, and 3 respectively). All criteria loaded positively on the first component ranging from .650 to .207 (M=.491). The analysis was then re-run but the number of components was restricted to three.

\section{--- INSERT TABLE 5 ABOUT HERE ---}

Table 5 shows the unrotated and rotated criteria loadings. When comparing the effect of rotation, eight out of ten criteria remained in the same component with only Lying and Bailout moving from Component 1 to Component 2. The unrotated PCA shows Component 1 had the largest loadings for Withdrawal (.650), Preoccupation (.626), Tolerance (.626), Chasing 
(.561), Lying (.543), Loss of Control (.525), and Bailout (.504). Component 2 had the largest loadings for Risked Relationships (.598) and Illegal Acts (.560) although Bailout in Component 2 (.483) was very close to the Component 1 score (.504). Component 3 was largest for Escape only (.795). The rotated PCA shows Component 1 had the largest loadings for Preoccupation (.766), Tolerance (.751), Withdrawal (.723), Loss of Control (.538), and Chasing (.484) (hypothesis 2 prediction; supported). Component 2 had the largest loadings for Risked Relationships (.731), Bailout (.696), Lying (.627), and Illegal Acts (.555). Component 3 had the largest loading for Escape only (.820). However, Loss of Control had roughly equivalent loadings for component 1 (.538) and component 2 (.464).

\section{Univariate Curves}

Figure 1 illustrates univariate curves for each DSM-IV criterion. On visual inspection, the Risked Relationships and Illegal Acts curves appear to have accelerating concave upwards curves, suggesting a 'high-threshold' relationship. Visual inspection found Loss of Control, Escape, Chasing, and Lying had decelerating concave downwards curves suggesting ‘lowthreshold' relationships. By comparison, Preoccupation, Tolerance, and Withdrawal appeared to be best described by a polynomial curve, while Bailout appeared to be described best by a linear curve. A second order polynomial (three free parameters) was also fitted for Bailout but reported the same goodness of fit as the linear curve so only the linear curve is reported in the interests of parsimony. 
Three criteria (Preoccupation, Withdrawal, and Tolerance) visually appeared to have curves that were both equally under- and over-predicted as compared to a linear curve. These criteria, although technically better described by polynomial curves, also approximated a linear relationship. Therefore, to quantify these effects, all curves were examined using the Akaike Information Criterion (AIC) model comparison analysis. When criteria were compared based on the best fitting polynomial or linear curves, linear curves were found to be superior (i.e. less information was lost compared to the 'true model' than the polynomial curves) than for any other curve type for all criteria (average AIC value, Linear 2.70, Polynomial 5.31: hypothesis 3 prediction; partially supported - although there was evidence for the continuum classification system only this taxonomy could be assessed using this analysis).

\section{Hierarchical Cluster Analyses}

Figure 2 is a dendrogram showing the average linkage between criteria and clusters for all 10 criteria. This shows the arrangement of clusters of criteria from the hierarchical cluster analysis where those with the most similarity join first (far left) and those that are most dissimilar join last (far right). The lengths of the horizontal lines between clusters indicate how close or similar these groups are to each other. Visually these clusters seem to coalesce into four clusters of increasing severity. Cluster one is the first to form consisting of Escape, Chasing, and Lying. Cluster two follows consisting of Preoccupation, Tolerance, Loss of Control, and Withdrawal, then cluster three consisting of Risked Relationships and Bailout, and finally cluster four consisting solely of Illegal Acts (hypothesis 2 prediction; supported, hypothesis 3 prediction; partially supported - although this analysis provides evidence of a hierarchy no other taxonomy could be assessed using this analysis). 
Gambling Disorder Hierarchy

\section{--- INSERT FIGURE 2 ABOUT HERE ---}

Although the groupings appear discrete, there appears to be a noticeable similarity in the agglomeration coefficients (indicated in Figure 2 by the horizontal distance between merges), namely the merging of Preoccupation and Tolerance appears to have a similar distance to the merge of Escape and Chasing, although the second pair to merge were part of cluster 2 and were formed before cluster 1 added Lying. This is supported in their agglomeration coefficients, where Escape and Chasing join at a distance of 1091 squared Euclidean values while Preoccupation and Tolerance join at 1131 although belonging to a separate cluster and forming immediately after Escape and Chasing merge.

\section{Discriminant Function Analyses}

A discriminant function analysis was used to predict the accuracy of different taxonomies based on the correspondence between models and the linear contributions of DSM-IV criteria. Examining the cut-off scores for optimal accuracy, the Toce-Gerstein et al. taxonomy was found to accurately predict $99.0 \%$ of the criteria classification using their stated numerical cut-offs (hypothesis 3 prediction; supported). Using the hierarchy of criteria produced by the cluster analysis rather than number of criteria endorsed (i.e., evidence of Illegal Acts was coded as the highest severity, evidence of Bailout or Risked Relationships resulted in the second highest severity, etc.), this taxonomy achieved a classification accuracy of 78.8\%. Examination of the classification results showed the majority of the classification errors were due to a significant group of highest severity gamblers mis-classified as the second 
highest severity gamblers. Consequently, when the highest Pathological category was subsumed into the second highest severity category (i.e., Illegal Acts join Risked Relationships and Bailout) to form three sub-groups, classification accuracy improved to 98.9\%. Finally, an assessment between DSM-IV and DSM-V systems (i.e. excluding Illegal Acts and using a 'case-ness' diagnosis between non-clinical vs. clinical disordered gambling) resulted in the DSM-IV cut-off score of five achieving 98.2\% predictive accuracy (hypothesis 3 prediction; supported) while the DSM-V cut-off score of four achieved a 96.5\% predictive accuracy.

\section{Discussion}

The current study investigated the frequency of individual DSM-IV criteria across gambling severities in a state-wide archival data set of gamblers presenting to specialist problem gambling services. Compared to the Toce-Gerstein et al. (2003) study, this study reports higher frequencies for all criteria consistent with previous research (Stinchfield, Govoni, \& Frisch, 2005).

Toce-Gerstein et al. (2003) concluded that dependence as measured by Withdrawal and Loss of Control best marked the threshold of meeting the diagnosis of pathological gambling. In the present study, all of the DSM-IV criteria, except Illegal Acts, differentiated between At Risk and Problem Gamblers, while all criteria were also significantly different between Problem, Low Pathological and High Pathological gambling, suggesting that the present study had clearer distinctions between subgroups than found in the Toce-Gerstein et al. (2003) study. 
Across the whole study, Escape emerged as the most frequently met criterion, with Chasing the second, where all criteria except for Illegal Acts were statistically associated with all other criteria. This is in contrast with Toce-Gerstein et al.’s (2003) community study, where Chasing was found to be the most frequently met criterion, and Escape third most frequent. The strongest association was between Preoccupation and Tolerance, indicating a correlation between cognitive and physiological symptoms. This association may provide evidence of a distinguishing characteristic of disordered gambling from the hallmark physiological symptoms in substance dependence, i.e. Tolerance and Withdrawal. Such knowledge may illustrate which disorder dominates for clients with dual gambling and substance disorders.

Within subgroups, the strongest associations appeared to also partially reflect the progression of gambling severity; Escape and Risked Relationships had the strongest associations for the At-Risk group, Lying and Lost Control for the Problem gambler group, Preoccupation and Risked Relationships for the Low Pathological group, and Withdrawal and Illegal Acts for the High Pathological group. Although Risked Relationships appears as a member of the strongest pair for the At-Risk and Low Pathological group, the appearance of Escape in the At-Risk group, and Illegal Acts in the High Pathological group, suggest that at the least and maximal severities, gamblers are experiencing the hallmarks of minimal and maximal harm.

Toce-Gerstein et al. (2003) argued that their hierarchy of disorders began with a common subclinical behaviour of Chasing gambling losses. In their study, Chasing was the most frequently met criterion when few other criteria were met (i.e., for At-Risk and Problem gamblers) or when many other criteria were met (Low and High Pathological gamblers). In the 
current study, Escape, Chasing, Lying, and Loss of Control were the most frequently met criteria for the entire sample and Escape was the most frequently met criterion for At-Risk, Problem, and Low Pathological gamblers. These results indicate that for all but the most disordered gamblers, Escape is the most commonly met criterion in a treatment-seeking sample and is consistent with previous research linking gambling with the motivation to avoid problems (Thomas, Sullivan, \& Allen, 2009). Similarly, gambling to Escape is often the precursor for further problems as subsequent inevitable gambling losses will compound the number of problems Escaping gamblers are seeking to remove themselves from by gambling.

A common gambling function was supported in the univariate curves where most criteria visually approximated a modest concave downwards curve, and all were more parsimoniously described by a linear curve. A linear curve can be thought of as representing a dependent relationship between the frequency of criterion endorsement and the total DSM-IV score. This result supports the continuum of risk understanding of gambling where increasing endorsement of criteria increases severity. Unfortunately, Toce-Gerstein et al. (2003) did not compare curves using a quantitative model comparison method so direct comparisons between studies about linearity using this approach are not possible.

The Principal Component Analysis showed three components; a primary component containing five criteria, a secondary component containing four, and a third component reporting a single criterion. These results are similar to other research, e.g., (Hodgins, 2004), but stand in contrast with Toce-Gerstein et al.(2003) in which a Principal Component Analysis found all criteria loaded on the first component except one. The implications for these results suggest the possibility of different gambling phenomenologies for treatment seekers compared to those in the community. In the current study, treatment seekers endorsed higher rates of 
criteria across the taxonomy as a whole especially to criteria analogous to the physiological hallmarks of substance dependence, Withdrawal and Tolerance. This implies treatment seekers are experiencing higher incidences of qualitatively different symptoms compared to the participants in the Toce-Gerstein et al. study. Moreover, all the criteria in component one reflect the major components of substance dependence (i.e., Tolerance, Withdrawal, and Loss of Control, while Preoccupation and Chasing are reflective of substance dependent user's persistent efforts to obtain and continue using despite their negative consequences), supporting the move to place disordered gambling within Substance Related and Addictive Disorders in the DSM-V rather than being part of the obsessive-compulsive disorder spectrum (Potenza, 2008). However, as disordered gambling itself involves no exogenous substances, these similarities are likely to be much less severe.

The cluster and discriminant analyses appeared to support the existence of a pathological gambling taxonomy similar to the one proposed by Toce-Gerstein. However, there was evidence for both a numerical cut-off and a criteria only hierarchy as both approaches produced similar, and very high, predictive accuracy scores. Further, the changes in the predictive accuracy were minimal when a cut-off score was reduced from five to four criteria, similar to previous reports (Stinchfield et al., 2005). These results raise the intriguing situation of multiple processes occurring simultaneously: a continuum of harm where increasing endorsement indicates increasing severity, a hierarchy of specific criteria across severities, and a dichotomous 'case-ness' of disordered gambling. The possibility of multiple simultaneous processes suggests that there are likely to be differences in the phenomenology of gambling as the number of criteria increase. Moreover, assuming a specific criteria hierarchical approach, the discriminant analyses indicate three phases of experience; initial use and development of a 
problem (Escape, Chasing, and Lying), problematic behavior (Loss of Control, Preoccupation, Withdrawal, and Tolerance), and the experience of negative consequences for others (Bailout, Risked Relationships, and Illegal Acts). This taxonomy implies a sub-clinical developmental phase where individuals engage in some experimentation (similar to initial drug use), a later stage meeting the current cut-off score for disordered gambling including the endorsement of the hallmark physiological symptoms of drug dependence (suggesting the possibility of a common neurological process for both disorders), and then in the most severe cases negative impacts on the associates of the gambler and ultimately the rest of society. Previous Australian reports suggest treatment seekers typically experience significant and persistent issues including substance use comorbidities, physical and psychological breakdowns, and a 'crisis' typically involving others that forces the gambler to seek treatment (Evans \& Delfrabbro, 2005; Productivity Commission, 2010). Our results reflect these findings and seem to indicate that treatment seekers are under significant individual and societal pressure to address their gambling. Hierarchical approaches may be useful for alerting gambling counselors to the potential of more serious issues if these higher severity criteria are endorsed even when clients report sub-clinical scores.

Additionally, in both the original study and the present study, Escape and Chasing were highly reported criteria. These apparent equivalent findings from different populations suggest that together Escape and Chasing criteria are likely to be a comprehensive basis for capturing subclinical and problematic gambling. Although the predictive ability of these items may interact with other variables, such as gender and age (Faregh \& Derevensky, 2011; Walker \& Shannon, 2006), their inclusion in a brief screening tool would probably improve screening sensitivity (Volberg, Munck, \& Petry, 2011). 
Compared to another treatment study (Hodgins, 2004), the current study had slightly more men, were on average younger, were less often born in the country where the study took place, and were more often not in a current relationship. This suggests that in the Australian context treatment seekers come from more disadvantaged groups; minorities and those without current relationships (i.e., having lower 'social capital' (Winter, 2000). This may imply, at least compared to the Hodgins study that Australian treatment seekers present as a more vulnerable population. Moreover, the high endorsement of Escape in the current study may be related to the high incidence of Electronic Gaming Machines (EGMs). Specifically, as there were higher frequencies of EGMs per person in Australia during the study period (Dowling, Smith, \& Thomas, 2005), and as this sample of treatment seekers nominated EGMs as their most frequent gambling activity (Jackson, Thomas, Holt, \& Thomason, 2005), and EGM play has been characterized as dissociative state inducing (McCormick, 2009), the higher frequencies of reporting gambling to Escape in the current study may be a result of the large numbers of EGMs in Australia and the ‘benefits' they ensue.

\section{Clinical Implications}

These results have practical implications for treatment providers. Clients who report the higher severity criteria are not only likely to experience more profound personal harms than gamblers who report only lower severity criteria, but their family and communities will also be experiencing significant harm. Therefore, treatment providers would be well advised, in addition to providing individual counseling to the gambler, to also provide financial counselling and guidance to the gambler’s family members and associates. Further, an important practice implication of the existence of a hierarchy is that if a client endorses fewer 
criteria than the cut-off score for 'case-ness', but endorses any high severity criteria (e.g., Bailout, Risked Relationships, and Illegal Acts), the clinician would be advised to probe further any unendorsed lower severity criteria as a hierarchy implies a high likelihood those lower severity criteria will also be in play.

\section{DSM V Implications}

The removal of the Illegal Acts criterion in the newly developed DSM-V is supported by the psychometric analyses reported in our large study of treatment seeking clients. Specifically, only High Pathological gamblers reported more than 15\% of their group as meeting the Illegal Acts criterion. Further, when Illegal Acts were excluded from numerical and criteria specific hierarchies, it had little effect on predictive accuracy. As well, similar to a recent report examining the then proposed changes for DSM-V pathological gambling diagnoses, predictive accuracy remained high when Illegal Acts was excluded from diagnosing pathological gambling 'case-ness' (endorsement of five or more criteria) and when the diagnostic cut-off score was reduced to four (Petry, Blanco, Stinchfield, \& Volberg, 2012).

Further, the evidence of the numerical hierarchy we found in our treatment sample is congruent with the numerical hierarchy reported in the DSM-V of mild (4-5), moderate (6-7), and severe (8-9) harms. This hierarchy indicates that the DSM-V gambling disorder work group also considers the gambling disorder to function at differing levels, perhaps opening the door for the possibility of different gambling phenomenologies similar to the hierarchy reported in this study.

\section{Strengths and Limitations}


There were a number of limitations with the present study. First, although specialist problem gambling clinicians all used the same criteria, there was the possibility that some may have used more questions than other clinicians to assess criteria. We also measured current DSM-IV criteria endorsement while the Toce-Gerstein et al. study reported life-time endorsement. Another possible weakness was that this study was cross-sectional so these analyses are limited to describing behavior of the clients at the time of the study and cannot make claims about any possible temporal effects, i.e. changes in gambling behavior for clients over time. Similarly, as this was a treatment seeking study, we cannot make judgments about the incidence of criteria in the general population. Further, we made comparisons between samples from two different countries where the Toce-Gerstein et al. study recruited participants using two different methods. These differences may also contribute to the differences between studies. Moreover, as the dataset reported no comorbid substance dependence or mood disorders, this is also a study limitation due the high comorbidity between substance dependence and disordered gambling. We also acknowledge that further analyses such as concurrent validity assessments are required before a disordered gambling hierarchy can be accepted.

However, this study also has strengths. It replicates most of the analyses of the ToceGerstein et al. (2003) study in a large sample of treatment-seeking gamblers, as suggested by Petry (2003) whilst finding new and potentially important results. Specifically, the results identified a new sub-clinical criterion, where the Principal Component Analyses, cluster analyses, and discriminant analyses found a convergence on physiological criteria similar to drug dependence. These results support the inclusion of pathological gambling as part of the drug dependence rubric in the just released DSM V. 


\section{Conclusion}

These results suggest that a hierarchy similar to the Toce-Gerstein et al. taxonomy is feasible and supports disordered gambling to be located within Substance Related and Addictive Disorders in the DSM-V. Moreover, given that we found evidence for multiple classification systems, it appears that a simplistic approach for categorizing disordered gambling is likely to miss the apparent complexity we have found in our data. Understanding the precise combinations and when to use these possibly competing models of disordered gambling will provide a more nuanced understanding of gambling severity and ultimately improve the accuracy of clinical diagnoses.

\section{Conflict of Interest}

The authors declare that they have no conflict of interest. 
Gambling Disorder Hierarchy

\section{References}

Akaike, H. (1974). A new look at the statistical model identification. IEEE Transactions on Automatic Control, 19, 716-723.

American Psychiatric Association. (1980). Diagnostic and Statistical Manual of Mental Disorders (3rd ed.). Washington DC: Author.

American Psychiatric Association. (2000). Diagnostic and Statistical Manual of Mental Disorders (4th Text Revision ed.). Washington DC: Author.

Cavedini, P., Riboldi, G., Keller, R., D'Annucci, A., \& Bellodi, L. (2002). Frontal lobe dysfunction in pathological gambling patients. Biological Psychiatry, 51, 334-341.

de Ruiter, M. B., Veltman, D. J., Goudriann, A. E., Oosterlaan, J., Sjoerds, Z., \& van den brink, W. (2009). Response perseveration and ventral prefrontal sensitivity to reward and punishment in male problem gamblers and smokers. Neuropsychopharmacology, 34, 1027-1038.

Dowling, N., Smith, D., \& Thomas, T. (2005). Electronic gaming machines: are they the 'crack-cocaine' of gambling? Addiction, 100, 33-45.

Evans, L., \& Delfrabbro, P. (2005). Motivators for change and barriers to help-seeking in Australian problem gamblers. Journal of Gambling Studies, 21, 133-155.

Faregh, N., \& Derevensky, J. (2011). A comparative latent class analysis of endorsement profiles of DSM-IV diagnostic criteria for problem gambling among adolescents from a community and a treatment sample. Addiction Research and Theory, 119, 323-333.

Ferris, J., \& Wynne, H. J. (2001). The Canadian Problem Gambling Index. Ottawa: Canadian Centre on Substance Abuse.

Goudriaan, A. E., Oosterlaan, J., de Beurs, E., \& van den Brink, W. (2006). Neurocognitive functions in pathological gambling: a comparison with alcohol dependence, Tourette syndrome and normal controls. Addiction, 101, 534-547.

Hodgins, D. C. (2004). Using the NORC DSM Screen for Gambling problems as an outcome measure for pathological gambling: psychometric evaluation. Addictive Behaviors, 29, 1685-1690.

Jackson, A. C., Thomas, S. A., Holt, T. A., \& Thomason, N. (2005). Change and continuity in a help-seeking gambling population: A five-year record. Journal of Gambling Issues, 13, 1-31.

Kushner, M. G., Abrams, K., Donahue, C., Thuras, P., Frost, R., \& Kim, S. W. (2007). Urge to gamble in problem gamblers exposed to a casino environment. Journal of Gambling Studies, 23, 121-132.

Ladouceur, R., Silvain, C., Boutin, C., \& Doucet, C. (1998). Understanding and treating the pathological gambler. Chichester: John Wiley \& Sons.

Lesieur, H. R. (2006). The South Oaks Gambling Screen (SOGS): A rebuttal to critics. Journal of Gambling Issues, 17, 1-16.

Lorains, F. K., Cowlishaw, S., \& Thomas, S. A. (2011). Prevalence of comorbid disorders in problem and pathological gambling: systematic review and meta-analysis of population surveys. Addiction, 106, 490-498.

McCormick, J. E. (2009). Individual vulnerability and dissociative-like experiences in regular and problem gamblers. (Master of Psychology (Clinical) and Doctor of Philosophy), University of Adelaide, Adelaide. 
Neal, P., Delfrabbro, P., \& O'Neil, M. (2005). Problem gambling and harm: Towards a national definition. Melbourne: Office of Gaming and Racing on behalf of Gambling Research Australia.

Orford, J., Wardle, H., Griffiths, M., Sproston, K., \& Erens, B. (2010). PGSI and DSM-IV in the 2007 British Gambling Prevalence Survey: Reliability, item response, factor structure and inter-scale agreement. International Gambling Studies(10), 31-44.

Petry, N. M. (2003). Moving beyond a dichotomous classifcation for gambling disorders. Addiction, 98, 1673-1679.

Petry, N. M., Blanco, C., Stinchfield, R., \& Volberg, R. A. (2012). An empirical evaluation of the proposed changes for gambling diagnosis in the DSM-5. Addiction, 108, 575-581.

Potenza, M. N. (2008). The neurobiology of pathological gambling and drug addiction: an overview and new findings. Philosophica Transactions of the Royal Society of Biological Sciences, 363, 3183-3189.

Productivity Commission. (2010). Gambling. Canberra: Author.

Shaffer, H. J., \& Martin, R. (2011). Disordered Gambling: Etilogy, Trajectory, and Clinical Considerations. Annual Review of Clinical Psychology, 7, 483-510.

Slutske, W. S., Eisen, S. A., True, W. R., Lyons, M. J., Goldberg, J., \& Tsuang, M. T. (2003). Common genetic vulnerability for pathological gambling and alcohol dependence in men. Archives of General Psychiatry, 57, 666-673.

Stinchfield, R., Govoni, R., \& Frisch, G. R. (2005). DSM-IV diagnostic criteria for pathological gambling: Reliability, validity, and classification accuracy. The American Journal on Addictions, 14, 73-82.

Strong, D. R., \& Kahler, C. W. (2007). Evaluation of the continuum of gambling problems using the DSM-IV. Addiction, 102, 713-721.

Thomas, A. C., Sullivan, G. B., \& Allen, F. C. L. (2009). A theoretical model of EGM problem gambling: More than a cognitive escape. International Journal of Mental Health and Addiction, 7, 97-107.

Toce-Gerstein, M., Gerstein, D. R., \& Volberg, R. A. (2003). A hierarchy of gambling disorders in the community. Addiction, 98, 1661-1672.

Volberg, R. A., Munck, I. M., \& Petry, N. M. (2011). A quick and simple screening method for pathological and problem gamblers in addiction programs and practices. American Journal on Addictions, 20, 220-227.

Walker, M., \& Shannon, K. (2006). Gender differences in meeting the DSM-IV criteria for pathological gambling. Gambling Research, 18, 33-41.

Welte, J., Barnes, G., Wieczorek, W., Tidwell, M. C., \& Parker, J. (2001). Alcohol and gambling pathology among U. S. adults. Prevalence, demographic patterns and comorbidity. Journal of Studies on Alcohol and Drugs, 62, 706-712.

Winter, I. (2000). Towards a theorised understanding of family life and social capital (Vol. 21). Melbourne: Australian Institute of Family Studies. 
Table 1. Proportion of participants meeting each DSM-IV criterion

\begin{tabular}{|c|c|c|c|c|}
\hline \multirow[t]{2}{*}{$\begin{array}{l}\text { Criterion } \\
\text { no. }\end{array}$} & \multirow[t]{2}{*}{ DSM-IV criteria } & \multicolumn{2}{|c|}{$\begin{array}{l}\text { Current sample } \\
(\mathrm{n}=4349)\end{array}$} & \multirow{2}{*}{$\begin{array}{l}\text { Toce-Gerstein et al. } 2003 \\
(\mathrm{n}=399) \\
\% *\end{array}$} \\
\hline & & $\mathbf{n}$ & $\%$ & \\
\hline 5. & Escape & 3793 & 87.2 & 33.6 \\
\hline 6. & Chasing & 3510 & 80.7 & 59.6 \\
\hline 7. & Lying & 3330 & 76.6 & 24.1 \\
\hline 3. & Lost Control & 3254 & 74.8 & 15.3 \\
\hline 1. & Preoccupation & 2716 & 62.5 & 38.8 \\
\hline 10. & Bailout & 2502 & 57.5 & 15.3 \\
\hline 4. & Withdrawal & 2467 & 56.7 & 18.0 \\
\hline 9. & Risked Relationships & 2426 & 55.8 & 15.5 \\
\hline 2. & Tolerance & 2393 & 55.0 & 16.0 \\
\hline 8. & Illegal Acts & 916 & 21.1 & 4.0 \\
\hline
\end{tabular}

* No count was reported in the Toce-Gerstein et al. study 
Table 2. Classification of participants meeting Toce-Gerstein et al. Taxonomy

\begin{tabular}{|c|c|c|c|c|}
\hline \multirow{3}{*}{$\begin{array}{l}\text { Toce-Gerstein et al. } 2003 \\
\text { Taxonomy }\end{array}$} & \multirow{2}{*}{\multicolumn{2}{|c|}{$\begin{array}{l}\text { Current study } \\
(n=4349)\end{array}$}} & \multicolumn{2}{|c|}{ Toce-Gerstein et al 2003} \\
\hline & & & \multicolumn{2}{|c|}{ study } \\
\hline & $\mathbf{n}$ & $\%$ & $\mathbf{n}$ & $\%$ \\
\hline At-Risk (1-2) & 286 & 7 & 278 & 70 \\
\hline Problem (3-4) & 701 & 16 & 58 & 15 \\
\hline Low Pathological (5-7) & 1918 & 44 & 45 & 11 \\
\hline High Pathological (8-10) & 1444 & 32 & 18 & 5 \\
\hline
\end{tabular}


Table 3. Proportion of participants reporting DSM-IV criteria using Toce-Gerstein et al. (2003) taxonomy

\section{DSM-IV Criteria Subgroup}

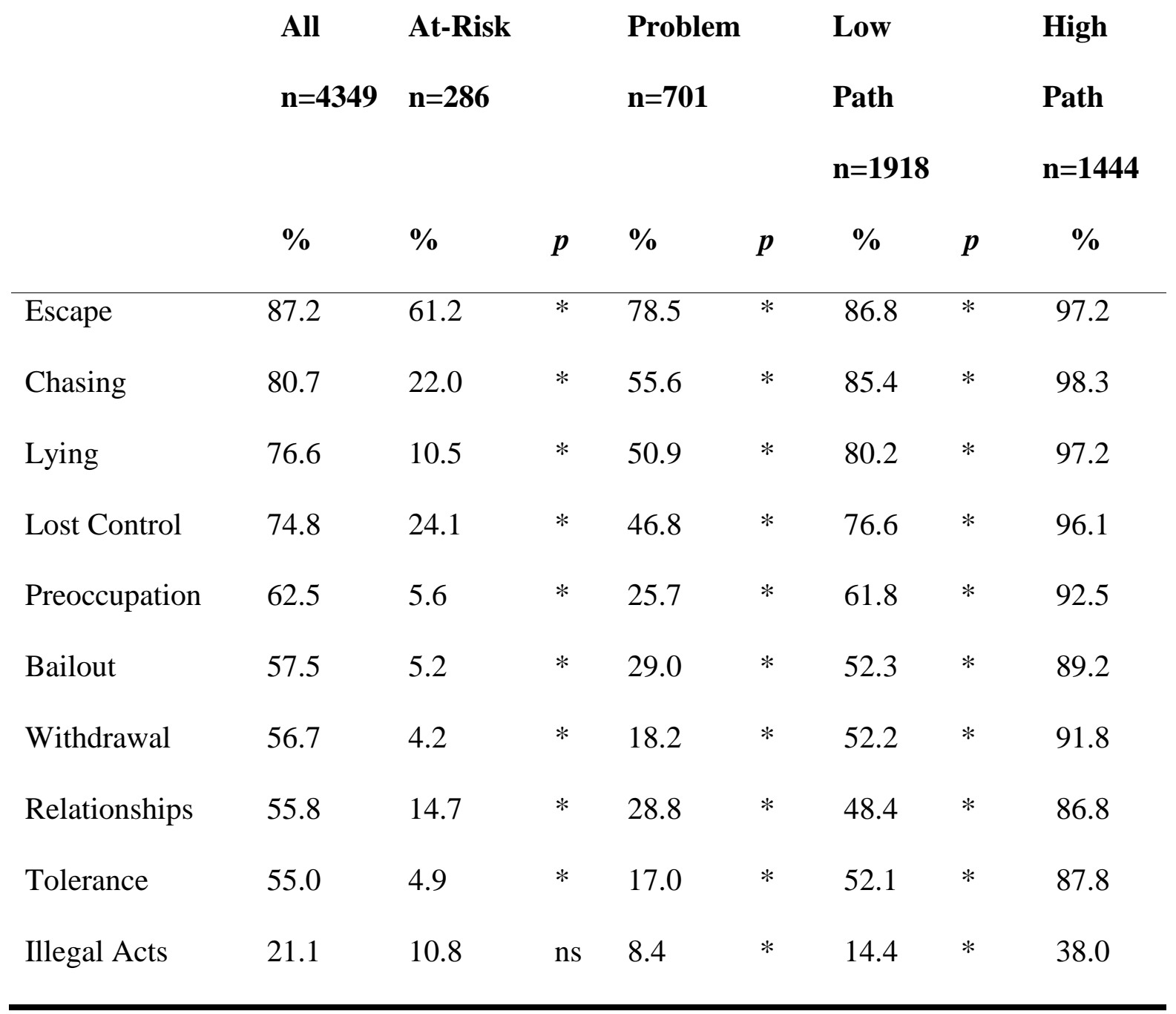

Note: Fisher's Exact tests, $*=p<0.001$, comparisons between adjacent subgroups across criteria.

Low Path $=$ Low Pathological

High Path = High pathological 
Table 4. Pair-wise Associations for the DSM-IV criteria for the entire sample

\section{DSM-IV Criteria}

\begin{tabular}{|c|c|c|c|c|c|c|c|c|c|c|}
\hline & 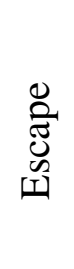 & 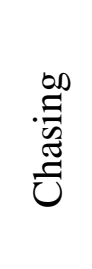 & 急 & 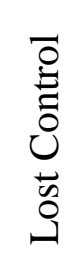 & 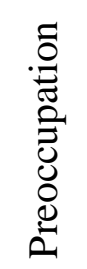 & 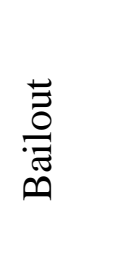 & 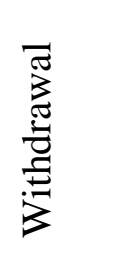 & 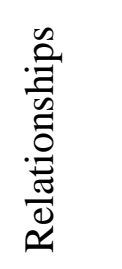 & 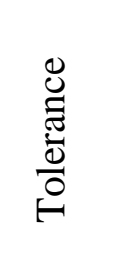 & 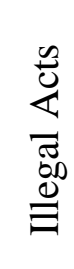 \\
\hline Escape & - & SS & ss & Ss & SS & ss & ss & SS & ss & SS \\
\hline Chasing & & - & ss & ss & ss & ss & ss & ss & ss & ss \\
\hline Lying & & & - & ss & ss & $.31^{* *}$ & ss & ss & ss & ss \\
\hline Lost Control & & & & - & ss & ss & $.41^{* *}$ & Ss & ss & ns \\
\hline Preoccupation & & & & & - & ss & $.41^{* *}$ & ss & $.47^{* *}$ & ss \\
\hline Bailout & & & & & & - & ss & $.32 * *$ & ss & SS \\
\hline Withdrawal & & & & & & & - & ss & $.36 * *$ & ns \\
\hline Relationships & & & & & & & & - & ss & ss \\
\hline Tolerance & & & & & & & & & - & ss \\
\hline Illegal Acts & & & & & & & & & & - \\
\hline
\end{tabular}

ns = non-significant association

ss $=$ significant association but smaller than .30

$* *=$ significant association $(\mathrm{p}<.001)$

- = identity 
Table 5. Unrotated and Rotated Component Loadings

\begin{tabular}{lrrrrrr}
\hline & \multicolumn{9}{c}{ Components } \\
DSM-IV Criteria & $\mathbf{1}$ & $\mathbf{2}$ & $\mathbf{3}$ & $\mathbf{1}$ & $\mathbf{2}$ & $\mathbf{3}$ \\
& & & & & & \\
\hline Preoccupation & .626 & -.357 & -.318 & .766 & .138 & -.004 \\
Tolerance & .626 & -.332 & -.300 & .751 & .158 & .007 \\
Loss of Control & .525 & -.276 & .248 & .538 & .139 & .464 \\
Withdrawal & .650 & -.356 & .030 & .723 & .158 & .323 \\
Escape & .241 & -.049 & .795 & .089 & .128 & .820 \\
Chasing & .561 & .020 & -.015 & .484 & .383 & .171 \\
Lying & .543 & .358 & .092 & .297 & .627 & .189 \\
Illegal & .207 & .560 & -.367 & -.004 & .555 & -.388 \\
Relationships & .426 & .598 & .013 & .102 & .731 & .025 \\
Bailout & .504 & .483 & .146 & .197 & .696 & .199 \\
\hline
\end{tabular}



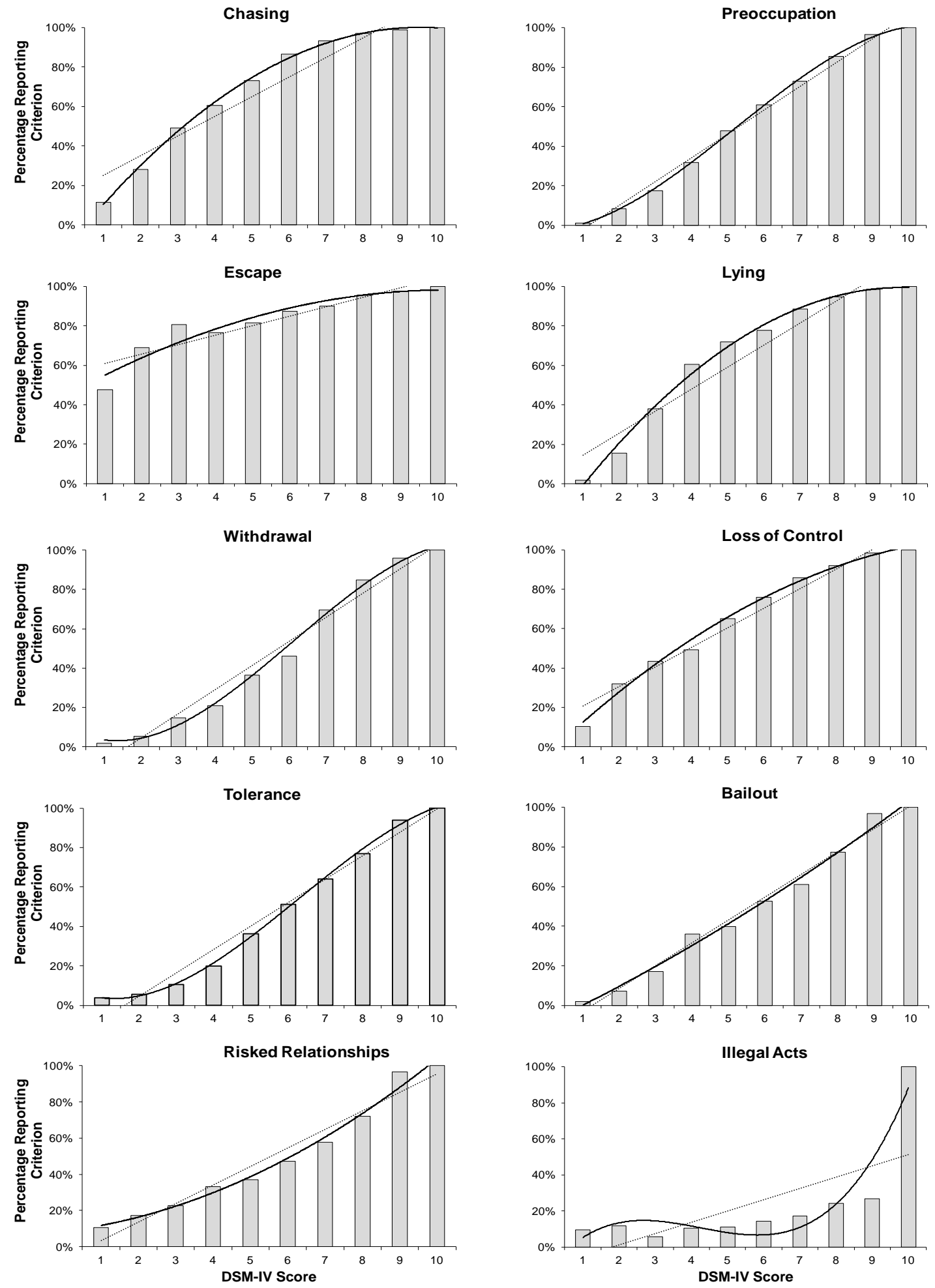

Figure 1. Univariate curves: Cumulative frequency of DSM-IV criteria*

*Solid line is best fitting polynomial, dotted line is linear regression. 
Average Rescaled Distance Between groups

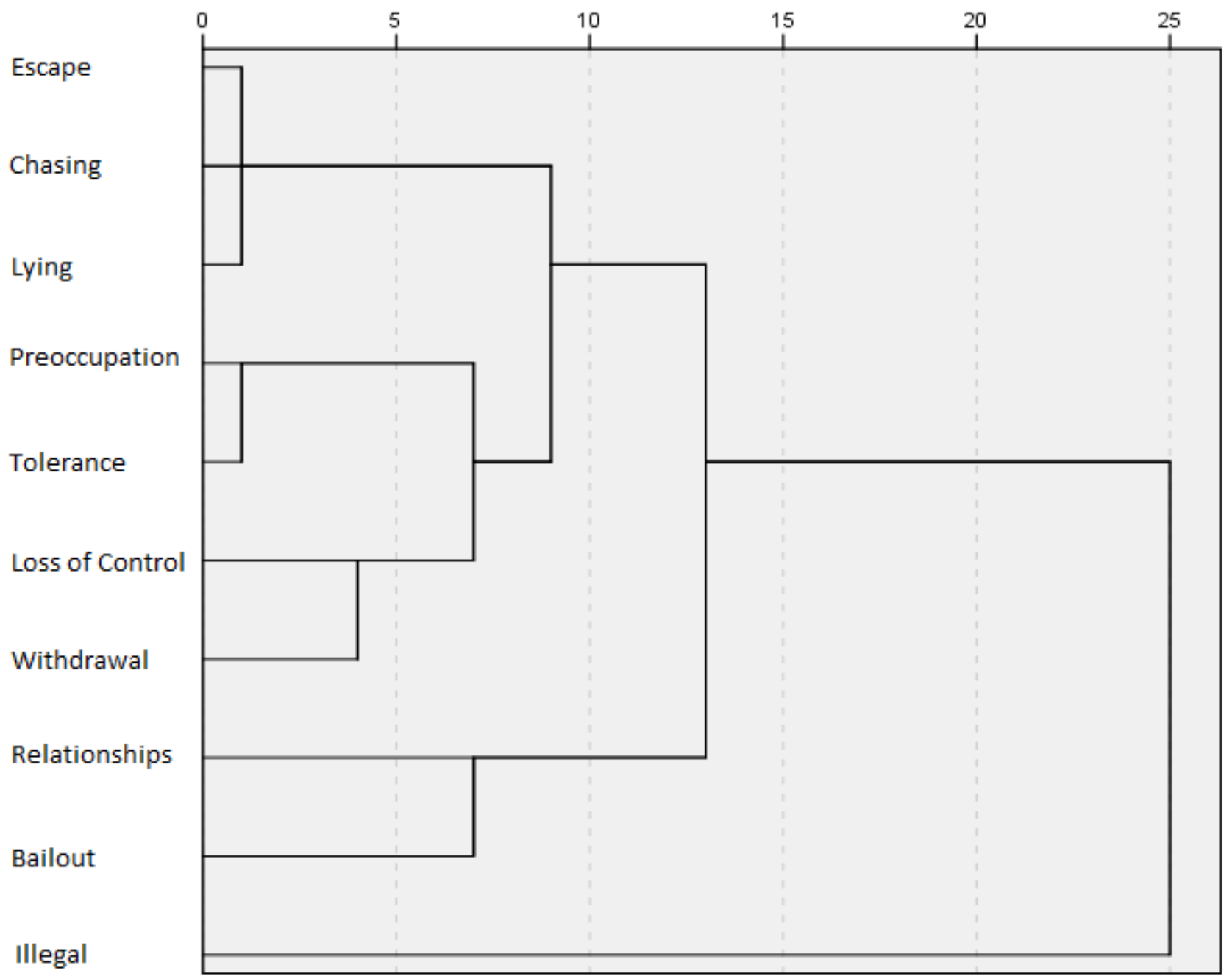

Figure 2. Dendrogram 